\section{El entrenamiento basado en la simulación como innovación imprescindible en la formación médica}

G. Vázquez-Mata a , A. Guillamet-Lloveras ${ }^{\text {b }}$
Cuestionario

de acreditación

www.educmed.net

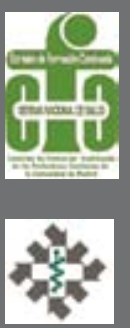

El entrenamiento basado en la simulación consiste en sustituir la realidad por un escenario simulado en el que estudiantes de medicina y profesionales pueden entrenar para adquirir habilidades de comunicación, psicomotrices o de trabajo en equipo. Dichos escenarios, y las metodologías que se aplican en ellos, varían según las habilidades a entrenar. Este tipo de entrenamiento va siempre asociado a una sesión de retroalimentación en el que participantes y tutores analizan la actividad realizada, sus puntos fuertes y los aspectos a mejorar; esta sesión se debe acompañar de una fase de pensamiento reflexivo y crítico, para profundizar en las ciencias básicas y clínicas del proceso entrenado. El empleo secuencial de diversos tipo de simulaciones puede utilizarse como circuito de entrenamiento o como prueba tipo examen clínico objetivo estructurado. En ambos casos, la evaluación es la última fase imprescindible de simulación aplicada a medicina. La simulación tiene una curva de aprendizaje excelente por su efectividad y rapidez, a la vez que aporta seguridad a los pacientes.

Palabras clave. Entrenamiento basado en la simulación. Formación médica. Simulaciones.

\section{Simulation-based training as an indispensable innovation in medical training}

Simulation-based training consists in replacing reality with a simulated scenario in which medical students and professionals can train in order to acquire communication, psychomotor or teamwork skills. Such scenarios, and the methodologies that are applied in them, vary according to the skills that are to be trained. This type of training is always linked to a feedback session in which participants and tutors analyse the activity that has been carried out, and discuss its strong points and other aspects that need improving. This session must be accompanied by a phase of reflective critical thinking in order to gain a deeper understanding of both the basic and the clinical sciences involved in the process being trained. The sequential utilisation of different types of simulations can be used as a training circuit or as an objective structured clinical examination-type test. In both cases, evaluation is the last essential phase of simulation applied to medicine. Simulation has an excellent learning curve due to its effectiveness and speed, while also increasing the level of safety for patients.

Key words. Medical training. Simulation-based training. Simulations.

\section{Competencias y componentes de la práctica clínica}

Con las palabras 'practica clínica' se indica el conjunto de actividades que un médico realiza en la cabecera del paciente. La competencia profesional de los médicos se ha definido como la practica clínica basada en el empleo juicioso del conocimiento médico y del razonamiento clínico, junto con habilidades técnicas y de comunicación, aplicados con actitudes positivas y valores; la competencia profesional permite a los médicos actuar beneficiando a los pacientes $y$ a la comunidad a la que pertenecen [1]. Esta definición engloba el conjunto de siete competencias básicas que se recogen en la nueva ley sobre requisitos de los planes de estudio para obtener el Titulo de Grado de Medicina, así como en las recomendaciones de diversas instituciones y sociedades científicas de la Unión Europea [2,3]. 
La construcción de la práctica clínica y su excelencia se inicia en la etapa de estudiante de medicina, se perfecciona con la etapa de especialización y continúa su desarrollo con la etapa de pleno ejercicio profesional. La práctica clínica supone la adición de capas sucesivas que pueden identificarse en cuatro eslabones complementarios y entrelazados:

- El primer eslabón es la construcción del conocimiento medico centrado en el estudio de las enfermedades, a través de las clases magistrales, conferencias y, especialmente, libros; escalonadamente se van sumando conocimientos de diversas disciplinas hasta llegar a adquirir una visión global de la enfermedad.

- El segundo eslabón es el hombre enfermo, sobre el que se recogen datos de diversa índole, se integran en hipótesis diagnósticas que engloban diversas enfermedades posibles, $y$ sobre las cuales se diseña un plan de trabajo para confirmarlas o desestimarlas, y tratarlas adecuadamente.

- El tercer eslabón es el escenario en el que se encuentra el paciente, como puede ser su domicilio, un servicio de urgencias o un quirófano. Cada escenario tiene unas connotaciones diferentes, tanto de conocimientos como de habilidades, pero además la relación médico/ paciente se amplía a otros personas, ya sean familiares o miembros del equipo de trabajo.

- El cuarto eslabón viene dado por el propio sistema sanitario en el que el médico desarrolla su actividad y por el que el paciente y sus familiares se moverán a lo largo de la enfermedad (p. ej., se inicia en el domicilio, se atiende en un servicio de urgencias, pasa a quirófano y luego a una sala de hospitalización, para ser finalmente referido a un centro de familia para su seguimiento). Durante este periplo, el paciente requiere no sólo calidad asistencial, sino también seguridad y autonomía en sus decisiones, debiendo el médico ofertar un alto nivel de profesionalidad junto con una excelente coordinación entre los diferentes niveles en los que será atendido [1].

El lector habrá rápidamente identificado dos aspectos relevantes de este esquema de capas superpuestas; el primero es que los libros que describen las enfermedades no son útiles para dar respuestas a las necesidades que se generan a partir del primer eslabón, y en segundo lugar, que las habilidades medicas que requiere la práctica clínica van mas allá del conocimiento sobre enfermedades.

\section{Una visión rápida a las competencias que requiere cada eslabón}

Clásicamente, el primer eslabón se centra en el conocimiento recogido en los libros de texto y en las lecciones magistrales para los estudiantes, y en revistas y libros de especialización para los profesionales en ejercicio. Este conocimiento requiere integrarlo, comprenderlo y razonarlo. La evaluación de este conocimiento se realiza mediante pruebas casi siempre escritas, aunque también pueden ser orales; dependiendo del objetivo que se busque, las pruebas se diseñan de una u otra forma, si bien la mayor parte de las pruebas son de tipo respuesta múltiple y suelen centrarse en la memorización de datos. El gran recambio en la formación en el periodo de grado es el aprendizaje basado en problemas, que sustituye la clase magistral centrada en el profesor por el taller centrado en el alumno activo [4].

El segundón eslabón requiere, desde el nivel de grado, unas competencias básicas centradas en habilidades de comunicación, tanto orales como de escritura (p. ej., diálogo con el enfermo para recoger y escribir la historia clínica), y habilidades psicomotoras, auditivas y visuales (p. ej., semiología y algunas habilidades manuales básicas, como suturas o inyecciones), junto con habilidades cognitivas para integrarlos y trazar un plan de trabajo [3]. A partir del periodo de especialización, se añaden conocimientos y habilidades especificas (p. ej., habilidades psicomotoras para la cirugía laparoscópica) y se debe perfeccionar la comunicación con el paciente (consentimiento informado, malas noticias...) [5]. Este eslabón se entrenaba clásicamente sobre el paciente; una serie de limitaciones que comentaremos más adelante han aconsejado sustituir este contacto inicial por un entrenamiento basado en la simulación, es decir, sustituir la realidad por escenarios simulados que la reproducen y cuya ventajas explicaremos. Otra vertiente de la simulación es su empleo para evaluar habilidades comunicativas y psicomotoras, constituyendo el eje del denominado 'examen clínico objetivo estructurado' (ECOE), sobre el que volveremos más adelante. 


\section{Programa de Formación Médica Acreditada en Educación Médica}

Artículo: El entrenamiento basado en la simulación como innovación...

Autores: G. Vázquez-Mata, et al

Revista: Educ Med 2009; 12: 149-55

1. Una de las aseveraciones que se citan no forma parte de las características básicas que definen de manera global la competencia profesional de los médicos, ¿cuál es?:

$\square$ a) aplicación juiciosa del conocimiento médico

$\square$ b) habilidades técnicas

$\square$ c) buena comunicación

$\square$ d) excelente accesibilidad

$\square$ e) valores

\section{La simulación reproduce la realidad en escenarios} controlados que permiten entrenar a alumnos y profesionales. Una de los siguientes tipos de simulación no es correcta, ¿puede identificarla?:

$\square$ a) maniquís humanos interactivos

$\square$ b) actores que simulan enfermedades

$\square$ c) juegos de rol

$\square$ d) libros interactivos online

$\square$ e) equipos de realidad virtual para cirugía laparoscopia

3. La simulación tiene todas las características que se citan menos una, ¿cuál?:

$\square$ a) una curva de aprendizaje lenta

$\square$ b) las habilidades aprendidas se transfieren a la realidad

$\square$ c) posibilita las repeticiones

$\square$ d) promueve la seguridad de los pacientes

$\square$ e) compensa la falta de tiempo libre

4. Para entrenar habilidades de comunicación, ¿qué tipo de simulación cree que se amolda mejor?:

$\square$ a) simulación con realidad virtual

$\square$ b) simulación con equipos de realidad virtual

$\square$ c) simulación con actores

$\square$ d) juegos de rol

$\square$ e) entrenamiento en el manejo de equipos básicos tipo ECG

5. En caso de iniciar una rotación por una UCl, ¿qué tipo de simulación se amolda más a las necesidades de aprendizaje en estas unidades?:

$\square$ a) simulación con modelos anatómicos parciales y no interactivos

$\square$ b) juegos de rol

$\square$ c) simulación con actores

$\square$ d) simulación con equipos virtuales

$\square$ e) simulación con maniquís humanos interactivos
6. Una de las características que ha de tener la práctica clínica es el análisis y la prevención de errores médicos. ¿Qué tipo de simulación cree que debe aplicarse para entrenar este tipo de actividad?

$\square$ a) simulación con actores

$\square$ b) simulación con modelos anatómicos parciales y no interactivos

$\square$ c) simulación con equipos virtuales

$\square$ d) simulación con maniquís humanos interactivos

$\square$ e) juegos de rol

7. Un tutor que trabaje en simulación es aconsejable que reúna las siguientes características menos una, identifíquela:

$\square$ a) ser accesible

$\square$ b) crear un clima positivo

$\square$ c) tener una escucha activa

$\square$ d) ser intrusivo

$\square$ e) generar preguntas

8. La simulación tiene que reunir una serie de características para que produzca los máximos beneficios educativos. ¿Existe alguna característica incorrecta en el listado siguiente?

$\square$ a) debe de realizarse con el máximo realismo

$\square$ b) se requiere siempre una prueba de evaluación

$\square$ c) puede prescindirse de la retroalimentación en ciertas ocasiones

$\square$ d) la simulación tiene que ser reproducible

$\square$ e) su diseño requiere muchas horas de trabajo

9. La simulación difiere de la clase magistral en todas sus dimensiones. En relación con esta aseveración, ¿qué respuesta es incorrecta?:

$\square$ a) la simulación se basa en la memorización

$\square$ b) en la simulación, el alumno es activo

$\square$ c) la simulación no requiere aulas

$\square$ d) el profesor pierde su rol central

$\square$ e) la simulación requiere guiones

10. El entrenamiento para trabajar en equipo se fija en los siguientes aspectos excepto en uno de ellos. ¿En cuál?:

$\square$ a) trabajo coordinado

$\square$ b) reparto de funciones

$\square$ c) liderazgo

$\square$ d) retroalimentación permanente al equipo

$\square$ e) habilidades individuales

Nombre y apellidos.

DNI . .

Dirección

Código postal

Localidad....

Provincia/país.

Teléfono.

E-mail

Encontrará este cuestionario en la web de la revista: www.educmed.net 
En el tercer eslabón, además de contextualizar más profundamente la formación de los especialistas en formación o ya en pleno ejercicio profesional (p. ej., entrenamiento en cirugía robótica a distancia), aparece una nueva dimensión, el trabajo en equipo. El éxito en cualquier actividad clínica y la seguridad del paciente se basan en una serie de premisas del trabajo en equipo, donde convergen profesiones y especialidades diferentes; la percepción de las situaciones que hay que afrontar, la anticipación de las acciones necesarias, la toma de decisiones que se ajusten al plan trazado, el manejo y distribución de las actividades a implementar en cada momento, el liderazgo del grupo, la escucha activa y la comunicación permanente entre todo el grupo son sus claves. El entrenamiento de los equipos debe realizarse en escenarios que remeden situación complejas donde la situación del paciente pueda variar con rapidez; como veremos, la robótica interactiva avanzada, que permite simular situaciones de crisis, o bien los quirófanos reales, donde se entrena con modelos de cadáveres humanos o animales, permiten entrenar equipos en todas las dimensiones simultáneas necesarias [6]. La evaluación sigue una serie de normas que la hacen objetiva y reproducible.

El último eslabón está emergiendo actualmente como una nueva dimensión insoslayable de la formación de grado y posgrado. La ética, el manejo de los recursos, la seguridad del paciente y las diversas dimensiones que requiere la profesionalidad de los médicos se están empezando a entrenar recurriendo a la simulación basada en juegos de rol (rol playing), en los que los participantes asumen diferentes papeles para aprender a manejar situaciones complejas como errores médicos, acciones de mejora, o solucionar dilemas éticos. El análisis de casos y el videoanálisis también son instrumentos de entrenamiento. La evaluación de este tipo de simulaciones está menos desarrollada, pero existe un consenso sobre las etapas que hay que seguir y el tipo de propuesta final que el grupo del taller debe presentar [7].

\section{El entrenamiento basado en la simulación como una necesidad imprescindible}

Actualmente existen una serie de circunstancias que aconsejan que el entrenamiento en simula- ción anteceda a las actividades en la cabecera del paciente [5]:

- La curva de aprendizaje de las habilidades se acorta por múltiples razones, destacando:

a) Poder repetir el entrenamiento tantas veces como sea necesario hasta adquirir las habilidades entrenadas.

b) Entrenar aspectos clínicos que en condiciones normales pueden requerir meses o años (p. ej., la semiología de los ruidos cardiacos puede adquirirse en pocas horas de trabajo con un maniquí que los reproduzca).

c) Las habilidades adquiridas mediante la simulación son transferibles a la realidad.

d) Las curvas de aprendizaje basadas en la simulación son mejores que las curvas basadas en el entrenamiento clásico, y esto convierte el entrenamiento basado en la simulación en la herramienta ideal para afrontar los retos de la educación.

- Aumenta la seguridad de los pacientes disminuyendo los errores médicos. El entrenamiento basado en la simulación permite corregir:

a) La falta de experiencia clínica.

b) Los fallos en la coordinación del equipo de profesionales.

- La presión económica sobre los profesionales de hospitales y centros de atención primaria, así como nuevas normas laborales, están repercutiendo negativamente en el patrón clásico de entrenamiento en la cabecera del paciente.

- Los profesionales disponen de menos tiempo libre para enseñar o reciclarse.

- La rapidez de las altas hospitalarias y las limitaciones de tiempo en las consultas dificulta el seguimiento de los pacientes.

- La libranza después de las guardias, junto a sus aspectos positivos, ha disminuido el tiempo útil al lado de los pacientes.

- La curva de aprendizaje basada en la simulación, por su perfil de rapidez y efectividad, permite afrontar la escasez de tiempo.

- Los derechos de los pacientes, que obligan a los profesionales a informarle de las actividades que se van a realizar y a aceptar su rechazo si así fuera.

- La necesidad de reciclaje permanente de los médicos para mantener su competencia adecuada a las demandas de su entorno. 


\section{Clasificación práctica del entrenamiento basado en la simulación}

El entrenamiento basado en la simulación se puede clasificar de varias maneras [8], pero desde un punto de vista práctico puede resumirse del siguiente modo:

\section{Entrenamiento individual}

En los tres niveles de complejidad descrito, el entrenamiento se realiza en cada grupo alumno por alumno, o médico por médico:

- Complejidad baja: se basa en modelos sencillos que permiten practicar habilidades básicas aisladas (desde aprender anatomía hasta ensayar habilidades elementales, como la intubación de la vía aérea).

- Complejidad intermedia: agrupa habilidades que ya requieren un nivel de integración entre sí. El paradigma es la adquisición de las habilidades clínicas, como es la historia y la exploración clínica, la formulación de aproximaciones diagnósticas y la escritura de prescripciones.

- Complejidad alta: se basa en el empleo de tecnologías de alta interactividad, es decir, que simulan la realidad, ofrecen información y requieren respuestas activas del profesional; permiten entrenar habilidades psicomotoras difíciles de adquirir, como los equipos de realidad virtual para entrenamiento de la endoscopia digestiva o de la cirugía laparoscópica, o bien el manejo y tratamiento de las arritmias.

\section{Entrenamiento en equipo}

Participan simultáneamente personas que pueden ser de especialidades o profesiones diferentes:

- Complejidad baja: maniquís humanos no interactivos o pasivos, como los que permiten entrenar todas las maniobras de reanimación cardiopulmonar, entrenadas aisladamente y conjuntarlas con este maniquí; otro ejemplo pueden ser las maniobras iniciales de extracción y estabilización de pacientes accidentados.

- Complejidad intermedia: talleres basados en juegos de rol, para el análisis de situaciones e incidentes adversos y errores médicos, así como de situaciones de mejora.
- Complejidad alta: tiene dos variantes; la primera se centra en el entrenamiento del equipo en situación de crisis (shock traumático, infarto de miocardio intraoperatorio...) y se recurre a maniquís humanos altamente interactivos que permiten reproducir las funciones cardiovasculares, respiratorias con una gran fidelidad, y todo esto dentro de escenarios de un gran realismo [9]; en esta simulación pueden participar simultáneamente intensivistas, médicos de urgencias, enfermeras, anestesistas y cirujanos. La segunda variante se centra en el entrenamiento quirúrgico avanzado que requiere la participación completa del equipo quirúrgico, como puede ser la cirugía robótica a distancia, que modifica todo el protocolo quirúrgico clásico [10].

En estos dos contextos señalados, entrenamiento individual y entrenamiento en equipo, y sus tres niveles de complejidad se puede recurrir a los siguientes tipos de simuladores:

\section{Simuladores de bajo perfil tecnológico, que representan partes del cuerpo humano}

Abarcan todo tipo de simuladores, desde la vía aérea y pulmones, hasta extremidades para practicar punciones, torsos para drenajes o punciones lumbares y cabezas para exámenes otoscópicos y oftalmológicos. La lista es interminable y se amplia permanentemente. En este apartado se incluye también el manejo de tecnologías de uso frecuente que el médico debe saber manejar, como los aparatos de electrocardiografía, de analítica seca, respiradores básicos de transporte o desfibriladores.

\section{Simuladores de alto perfil tecnológico, con interactividad maniquí/alumno-profesional}

Pueden reproducir alguna función concreta (sonidos cardiacos normales y patológicos) o bien varias de ellas globalmente (función cardiovascular y respiratoria); estas funciones se pueden programar mediante un software para simular un síndrome clínico seleccionado, que podrá ser susceptible de evoluciones diferentes, según las actuaciones que el equipo que entrene implemente. Dicho equipo, además de identificar el síndrome al que se enfrenta, tendrá que decidir el tipo de monitorización, las vías de perfusión, la necesidad de intubación y ventilación, 
la colocación de drenajes si fueran necesarios, las pruebas complementarias a pedir y decidir, y el tratamiento y la fluidoterapia más adecuados. Cada episodio de entrenamiento suele tener una duración máxima de 30 minutos y durante este tiempo el equipo deberá estar atento a la evolución del paciente para implementar nuevas medidas o bien corregir las existentes. Durante toda la simulación, el trabajo coordinado del equipo, el reparto de funciones, el liderazgo, la escucha activa y la retroalimentación serán tan importantes como las medidas clínicas que se adopten [11].

\section{Simuladores virtuales}

Para el entrenamiento de habilidades complejas, como la broncoscopia o la navegación e intervencionismo intravascular. Constan de tres partes: un equipo similar al de la técnica a entrenar (p. ej., broncoscopio o catéteres para navegación endovascular intervencionista), que se maneja en condiciones de cuasi realidad gracias a un software que reproduce las condiciones hápticas del manejo del equipo, a la vez que en una pantalla se visualiza la progresión de la prueba en las mismas condiciones que en la realidad.

\section{Simuladores y simulación en cirugía}

Este campo presenta algunas variantes con respecto a las descritas anteriormente. En primer lugar existe una gama que abarca desde equipos tipo caja transparente (pelvi trainers), que permiten aprender la manipulación básica de los sticks de cirugía laparoscópica, hasta equipos de realidad virtual con gran hapticidad para entrenar procedimientos quirúrgicos reglados.

Sin embargo, el gran empuje en la simulación quirúrgica viene dado por la reproducción completa de un quirófano, en el que utilizándose modelos anatómicos humanos o bien animales de experimentación, se entrenan procedimientos completos o situaciones quirúrgicas no previstas [6], y también los nuevos roles de modalidades quirúrgicas como la cirugía robótica.

\section{Simulación con actores}

Requiere entrenar a profesionales para que simulen enfermedades, también se pueden utilizar pacientes que voluntariamente se presten a ello. Esta variante de simulación permite desarrollar diferentes vertientes de la comunicación, como la petición del consentimiento informado, la comunicación de malas noticias o el diálogo con pacientes agresivos o familiares intrusivos.

\section{Simulación con juegos de rol [7]}

En esta variante de simulación son los propios alumnos o profesionales los que asimilan un papel y actúan conforme a él. Se utiliza para aprender la metodología de análisis de errores por equipos multidisciplinares o para diseñar acciones de mejora de la calidad.

\section{Otras simulaciones}

El empleo de portales de Internet para el desarrollo de cursos online es actualmente una realidad del día a día [12], pero el campo se va ampliando progresivamente con 'e-pacientes' simulados o con incipientes videojuegos que remedan desde pacientes aislados hasta el manejo de recursos en caso de catástrofes. El desarrollo de este tema requeriría un espacio que va más allá de los objetivos de este trabajo.

Cada habilidad a entrenar tiene su técnica más apropiada, debiéndose escalar desde las competencias básicas hasta las competencias de mayor complejidad; muchas veces se requerirá un entrenamiento en el que se mezclen diferentes técnicas.

\section{Puntos insustituibles de la simulación}

Existen una serie de normas de las que depende el acierto en el manejo del entrenamiento basado en la simulación [9-11,13,14]:

- La simulación debe basarse en un guión que refleje claramente la situación que se va a entrenar, los objetivos que se buscan y las competencias que se van a adquirir. La implementación de estos guiones estará bien sistematizada.

- Los listados de actividades que deben realizar los participantes servirán para la retroalimentación de la acción de entrenamiento.

- La retroalimentación es una de las partes imprescindibles de la simulación. Los propios participantes y el tutor analizan los puntos fuertes del grupo y los aspectos a reforzar; se 
utilizan los listados citados, el videoanálisis y la opinión de observadores expertos.

- El pensamiento reflexivo y crítico sobre el entrenamiento realizado debe complementar la retroalimentación, para ir más allá de un acto puramente mecanicista.

- La evaluación debe realizarse siempre; para que sea correcta, la simulación ha de tener criterios de validez y reproducibilidad para asegurar que cada grupo entrena las mismas competencias. Este criterio es muy importante cuando la evaluación es acreditativa.

- Los instrumentos de evaluación deben estar bien calibrados conteniendo todas las características claves a evaluar. Frecuentemente son listados de actividades (como se citó anteriormente), que suelen concluir con conceptos de tipo 'apto' o 'no apto'.

- Las simulaciones se pueden ordenar de manera secuencial en forma de circuito. Estos circuitos tienen unas normas bien establecidas, tanto si se utilizan como circuito de entrenamiento o como circuito para ECOE.

- El entrenamiento basado en la simulación debe quedar reflejado en el currículo de grado, en el de especialización y en el de formación continua.

- El entrenamiento basado en la simulación consume tiempo para prepararla y ejecutarla; el papel de profesor desaparece y se sustituye por el de tutor.

- La preparación de los tutores no es intuitiva, requiere entrenamiento. Las características del tutor [13] en este campo se pueden resumir en: gustarle la docencia, tener conocimiento y recursos docentes, ser accesible, crear un clima positivo, tener una escucha activa, no ser intrusivo, generar preguntas y promover el aprender a aprender.

El entrenamiento basado en la simulación, junto con el aprendizaje basado en problemas y la evaluación basada en ECOE y en el empleo de los portfolios, junto con el basado en portales de Internet interactivos, constituyen la arquitectura de la renovación educativa en medicina. El entrenamiento basado en la simulación permite soslayar las dificultades de toda índole que actualmente tiene la formación inicial en la cabecera de los pacientes, tanto por la rapidez y eficacia de sus curvas de aprendizaje, como la seguridad que supone para los pacientes. La lección pendiente en este momento es la creación de un número suficiente de laboratorios de simulación, tanto en facultades de medicina como en hospitales, que permitan el acceso de los profesionales a este tipo de entrenamiento, a la vez que se reconoce que la dedicación de los tutores al entrenamiento basado en la simulación requiere tiempo y reconocimiento por parte de las autoridades docentes y sanitarias.

\section{Bibliografía}

1. Pardell-Alentá $\mathrm{H}$, ed. El médico del futuro. Barcelona: Fundación Educación Médica; 2009.

2. BOE, Ministerio de Educación y Ciencia, Orden ECI 332/2008, de 13 de febrero.

3. Palsson R, Kellet J, Lindgren S, Merino S, Semple C, Serini D. Core competencies of the European internist: a discussion paper. Eur J Intern Med 2007; 18: 104-8.

4. Guillamet-Lloveras A, González-Carrión P, Heierle-Valero C, Celma-Vicente $\mathrm{M}$. El aprendizaje basado en problemas. Nursing 2008; 26: 64-6.

5. Vázquez G, Guillamet A, Chaves J. La simulación como herramienta de aprendizaje. DPM 2008; 1: 5-12.

6. Vázquez-Mata G, Ruiz-Castillo J. El futuro pasa por el entrenamiento médico y quirúrgico basado en la simulación. Cir Esp 2009; 86: 1-2.

7. National Patient Safety Agency. Root cause analysis. URL: http://www.npsa.nhs.uk/nrls/improvingpatientsafety. [14.09.2009].

8. Bradley P. The history of simulation in medical education and possible future directions. Med Educ 2006; 40: 254-62.

9. Issenberg B, McGaghie W, Petrusa E, Gordon D, Scalese R. Features and uses of high fidelity medical simulations that lead to effective learning. Systematic review. BEME Guide n. ${ }^{\circ}$ 4; 2004.

10. Vázquez-Mata G, Ruiz-Castillo J. El entrenamiento quirúrgico basado en la simulación, ¿̇una moda o una necesidad? Endosurgery SECLA 2009; 27: 1-3.

11. Moorthy K, Munz Y, Adams S, Pandey V, Darzi A. A human factor analysis of technical and team skills among surgical trainees during procedural simulations in a simulated operating room. Ann Surg 2005; 242: 631-9.

12. García-Aguilera F, ed. Guía de innovación metodológica en e.learning. Programa Espacio Virtual de Aprendizaje. Red de Espacios Tecnológicos de Andalucía; 2008.

13. Ramani S, Leinster $S$. Teaching in the clinical environment. Med Teach 2008; 30: 347-64.

14. Hammick M, Olckers L, Campion-Smith CH. Learning in interprofessional teams. Med Teach 2009; 31: 1-12. 
